\title{
NATURAL NANOPARTICLE STRUCTURE, PROPERTIES AND REACTIVITY FROM X-RAY STUDIES
}

\author{
G. A. Waychunas \\ Lawrence Berkeley National Laboratory, Earth Sciences Division
}

\begin{abstract}
Synthetic analogs of naturally occurring nanoparticles have been studied by a range of $\mathrm{x}$-ray techniques to determine their structure and chemistry, and relate these to their novel chemical properties and physical behavior. $\mathrm{ZnS}$ nanoparticles, formed in large concentrations naturally by microbial action, have an interesting core-shell structure with a highly distorted and strained outer layer. The strain propagates through the particles and produces unusual stiffness, but can be relieved by changing the nature of the surface ligand binding. Weaker bound ligands allow high surface distortion, but strongly bound ligands relax this structure and reduce overall strain. Only small amounts of ligand exchange causes transformations from the strained to relaxed state. Most remarkably, minor point contacts between strained nanoparticles also relaxes the strain. In $\mathrm{TiO}_{2}$ nanoparticles a similar core-shell structure is observed with distortions severe enough to produce an almost amorphous structure, perhaps similar to a silica glass. Fe oxyhydroxide nanoparticles appear to go through structural transformations dependent on their size and formation conditions, and display a crystallographically-oriented form of aggregation at the nanoscale that alters growth kinetics. At least one Fe oxyhydroxide mineral may only be stable on the nanoscale, and nonstoichiometry observed on the hematite surface suggests that for this phase and possibly other natural metal oxides chemistry may be size-dependent. Numerous questions exist on nanominerals formed in acid mine drainage sites, and by reactions at interfaces.
\end{abstract}

\section{INTRODUCTION}

Mineral nanoparticles are formed by a variety of natural processes, and are ubiquitous in the environment. Inorganic production occurs in acid mine drainage sites, where $\mathrm{Fe}^{2+}$-rich mine waters strike oxygenated waters, forming $\mathrm{Fe}$ oxyhydroxide nanoparticles by hydrolysis. Weathering processes extract metals and anions from rocks that may react at the surface to form nanoparticles that may enter streams, be taken off by wind, or aggregate locally. Anthropogenic processes such as coal burning in power plants has been shown to produce $\mathrm{UO}_{2}$ nanoparticles, besides a host of varied carbon and sulfide particulates. However, organic formation processes, mainly microbial, and indirect biological processes, lead to the largest concentrations of natural nanoparticles including $\mathrm{ZnS}, \mathrm{Fe}_{3} \mathrm{O}_{4}$, many metal sulfides, manganese minerals, oxalate minerals, and even nanoparticulate gold. Many massive rock units, such as limestones, had origins through biologically controlled precipitation on the nanoscale (Ehrlich, 1996). Microbial formation of nanoparticles may be a result of the organism's metabolic processes, i.e. the use of a soluble species like $\mathrm{Fe}^{2+}$ for energy acquisition, with release of insoluble $\mathrm{Fe}^{3+}$; from the action of an organism to shield itself from a toxin; or from ancillary processes related to the chemistry near the organism cell wall and biopolymer exudates (Banfield, 2001). Such nanoparticles are 
part of a natural (and anthropogenically affected) environmental complex within which we live, and hence are obvious candidates for study both to determine possible hazardous properties, but also to understand better the nature of oceanic, landscape and global geochemical cycles.

\section{DIFFERENCES BETWEEN NATURAL (INCLUDING SYNTHETIC ANALOG ) AND ENGINEERED NANOPARTICLES}

Compared to engineered nanoparticles, such as carbon nanotubes, natural nanoparticles differ in several ways. They are always formed in a hydrous environment, and so water and hydroxyl groups are common constituents; can be highly monodispersed due to natural size-selection processes; may have very complex shapes due to aggregation behavior; are generally not passivated, i.e. do not have stabilizing or encapsulating ligands bound to their surfaces; are extremely abundant (i.e. in every inhalation and in all drinking water); and have general low toxicity. These aspects lead to a series of interesting properties, some shared with engineered nanoparticles, including high surface area, high surface strain and disorder, unusual phase transformations, nonstoichiometric surfaces, unusual defect stabilization, and crystallographically-controlled aggregation. The key area of interest for our group is the relationship of physical properties to atomic scale structure and detailed chemistry.

\section{STRAINED ZnS NANOPARTICLES}

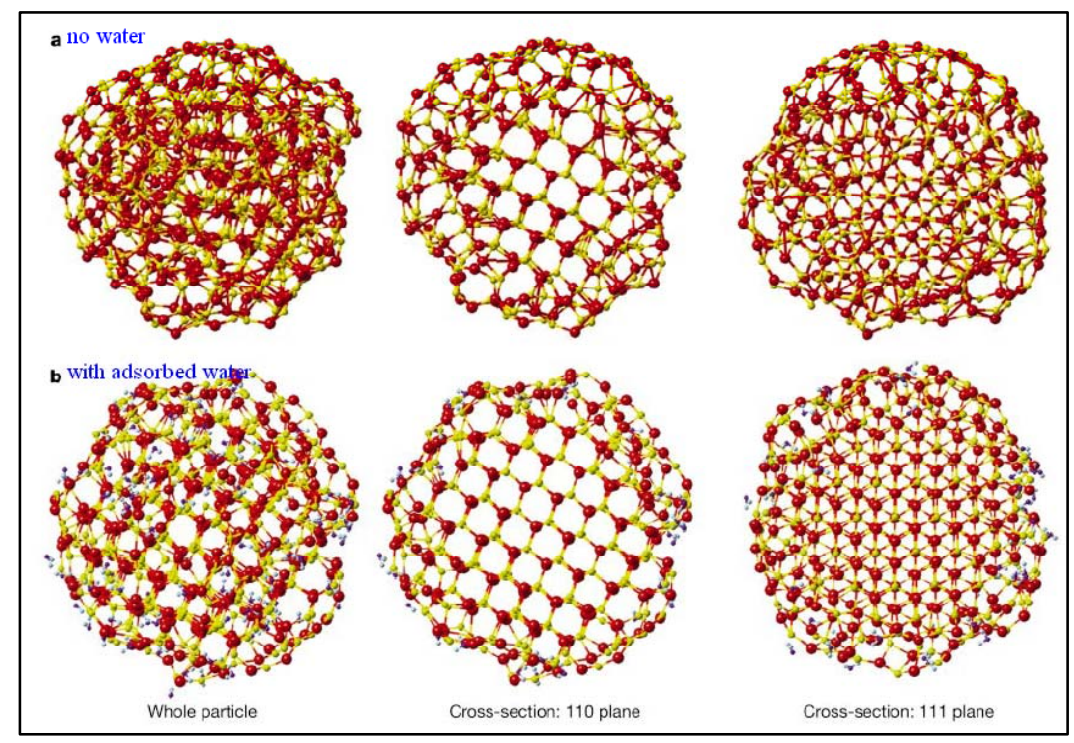

Figure 1. MD simulations of $\mathrm{ZnS}$ nanoparticles with and without water ligands. Without water, and also as formed in methanol, the structures are extremely distorted. The cross sections show quite irregular topology at the surface which penetrates well into the core. With the addition of a minimal layer of surface water this strained surface region collapses, though still present, and there is minimal strain into the core of the particle. 
Molecular Dynamics (MD) simulations of $\mathrm{ZnS}$ nanoparticles verified results obtained from XRD and pair distribution function (PDF) analysis (Figures 1 and 2). Synthesis in vacuum, or in a weakly bonding solvent like methanol leads to high distortion owing to dissatisfaction of surface bonding requirements. The effect of this strain can be seen in the PDF when compared to a

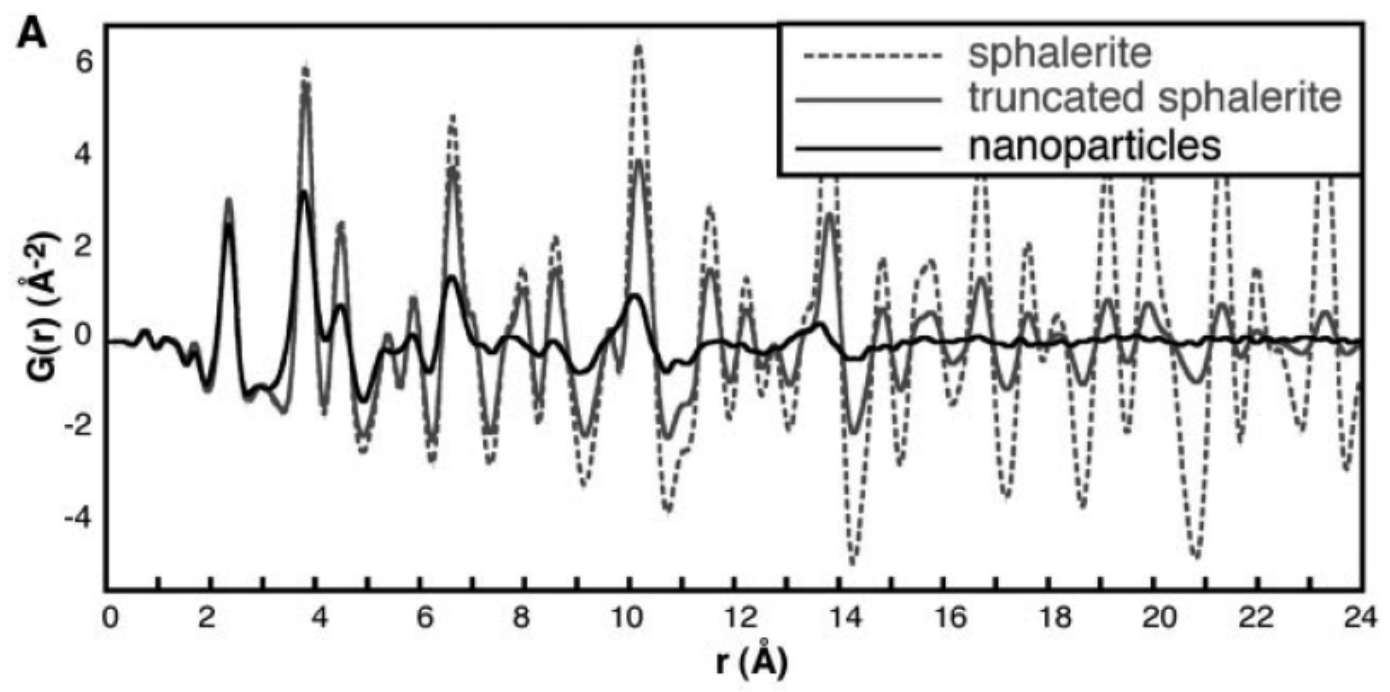

Figure 2. Calculated pair distribution functions (PDFs) for bulk sphalerite (cubic $\mathrm{ZnS}$ ) (dashed line); a $3.4 \mathrm{~nm}$ diameter sphalerite nanoparticle cut from the bulk structure with no relaxation (grey line); observed $\mathrm{ZnS}$ nanoparticles synthesized in mercaptoethanol. After Gilbert et al., 2004.

nanoparticle structure derived by cutting out a $3.4 \mathrm{~nm}$ sphere from bulk $\mathrm{ZnS}$ with no atomic positional relaxation. The "truncated" nanoparticle shows the effect of a decreasing number of pair correlations with increasing distance as expected, but there are still well-defined correlations at $2.4 \mathrm{~nm}$. In contrast the $3.4 \mathrm{~nm}$ experimental nanoparticles have highly damped correlations which are near zero beyond about $1.8 \mathrm{~nm}$. Analysis of the PDF in detail (Gilbert et al., 2004) shows that the two separate disorder effects are required to explain the data in addition to a lattice contraction: a thermal-like effect that produces random positional disorder, and a straindriven effect that increases as a function of interatomic pair distance (Figure 3). The effect of the strain on elastic properties can be further characterized by EXAFS analysis of the Zn-S first coordination shell as a function of temperature. When compared to bulk sphalerite, the nanoparticle shell is considerably stiffer, and fitting of an anharmonic Einstein oscillator model to the EXAFS reveals a 50\% higher Einstein frequency.

\section{EFFECT OF VARIED LIGAND BINDING AND AGGREGATION}

The strain at the surface of the $\mathrm{ZnS}$ nanoparticles depends on the type of ligand binding, and decreases progressively with the ligand-zinc binding enthalpy. This is shown in Figure 4 by a series of experimental PDF measurements for ligands with different binding energy. For convenience the ligands can be grouped into three different kinds, depending on the energy of the bond, the overlap orbital density of the ligand with the surface, and the inverse of the ligand- 
metal bond distance. The weakest ligand bonds result in the longest interatomic distances, least overlap, and highest distortion, hence their PDFs show the least order (Group I). In contrast, more strongly bonded ligands have the highest binding energy and the shortest interatomic distances, as well as the most ordered PDF patterns (Group III).

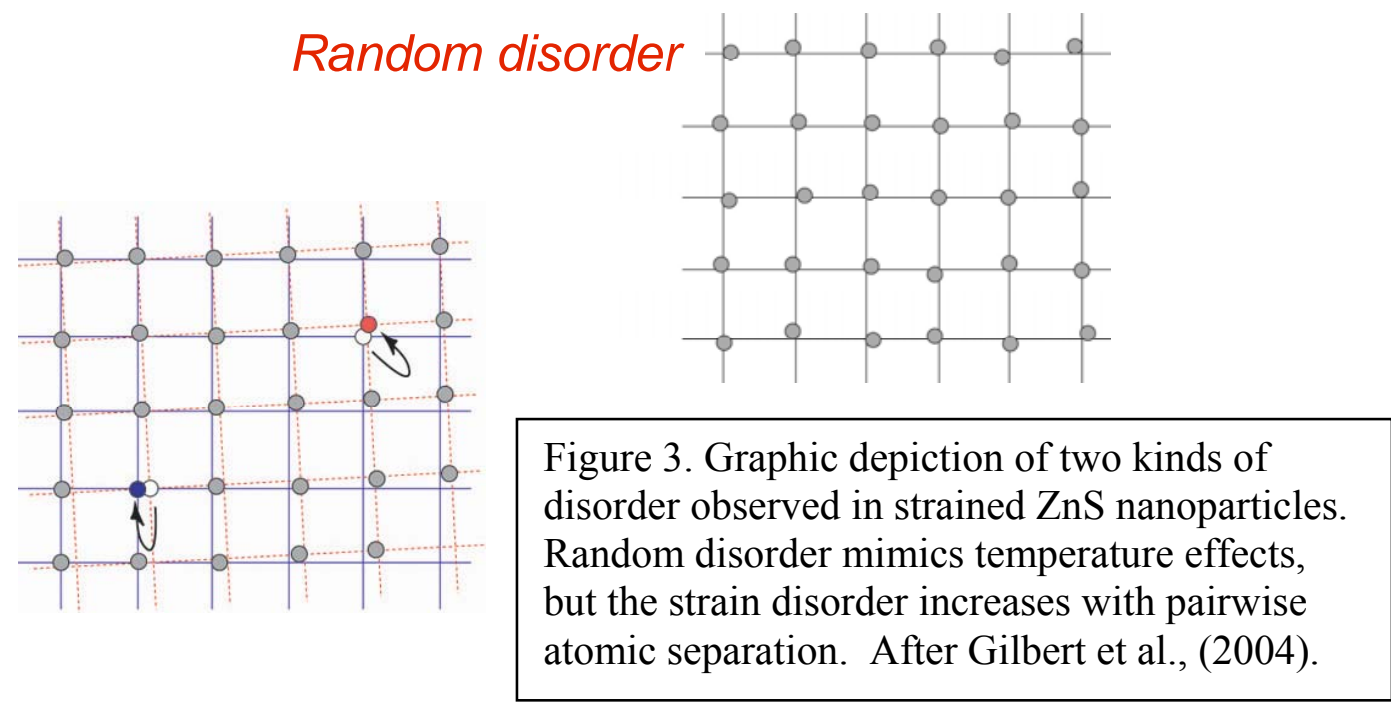

A surprising result of studies on $\mathrm{ZnS}$ nanoparticles was the analogous effect of aggregation and ligand binding. If synthesized in a weakly binding ligand the strained particles were observed to relax if permitted to aggregate (Huang et al., 2004). The transformation between the strained and relaxed states was reversible by sonication, and could be cycled many times. MD simulation of the effect verified the mechanism, as bond relaxation at only a few contact points between nanoparticles was enough to create the relaxation effect. Related studies of the effect of water concentration on the relaxation of $\mathrm{ZnS}$ formed in methanol, showed that only 5\% substitution for methanol created the relaxation. This verified that a relatively small number of surface binding

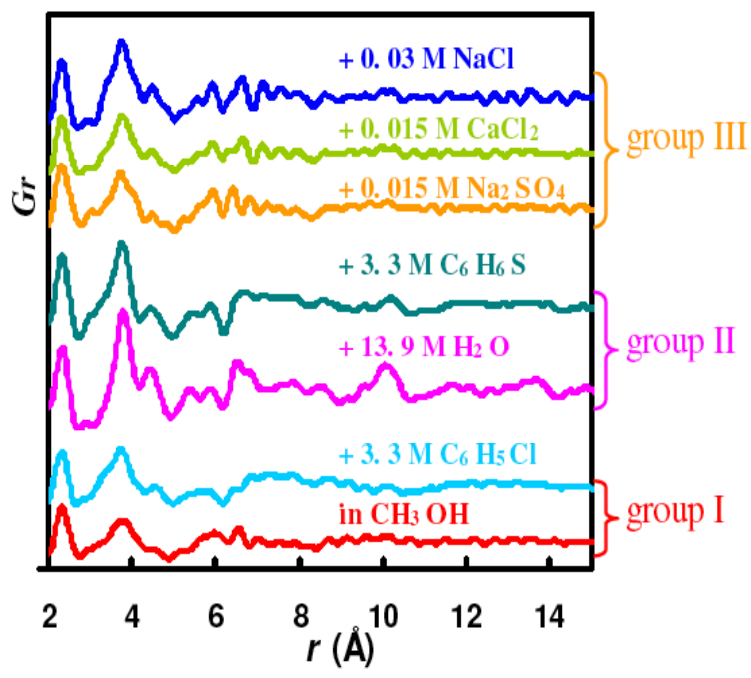

Figure 4. Changes in the PDF of 2 $\mathrm{nm} \mathrm{ZnS}$ nanoparticles as a function of ligand interactions. Group I shows the least structure and is consistent with weak ligand binding; group II shows intermediate structure and has intermediate binding energies; group III ligands are strongly bound creating maximal relaxation of strain. After Zhang et al., (2009). 
sites need to be affected to drive the transformation over the entire nanoparticle surface, consistent with the small number of attachment points in the aggregation MD simulations.

\section{Nonstoichiometry and unusual defects in Fe oxyhydroxide nanoparticles}

Figure 5 shows the relative numbers of oxygen types in an $\alpha$-FeOOH (goethite) nanoparticle as a function of diameter. Within the bulk, oxygen is coordinated by three Fe atoms, and Fe by six oxygens, yielding the $\mathrm{FeO}_{2}$ stoichiometry. To balance charge one of the oxygens is protonated. At the surface, oxygens have less Fe neighbors, and for a diameter of about $2 \mathrm{~nm}$ all three types of $\mathrm{O}-\mathrm{Fe}$ coordinations have equal proportions. In this sense alone small nanoparticles are nonstoichiometric as the oxygen concentration (or water content) increases with decreasing size, a fact borne out by recent calorimetry experiments (Navrotsky, 2008). This dependence on size is shown in the electronic structure on the $\mathrm{O} \mathrm{K}$ absorption edge, as a shift in the band positions, and change in band contribution with nanoparticle size, although some of the effect may be due to strain or relaxation. All nanogeothite was synthesized with water ligands, where strain
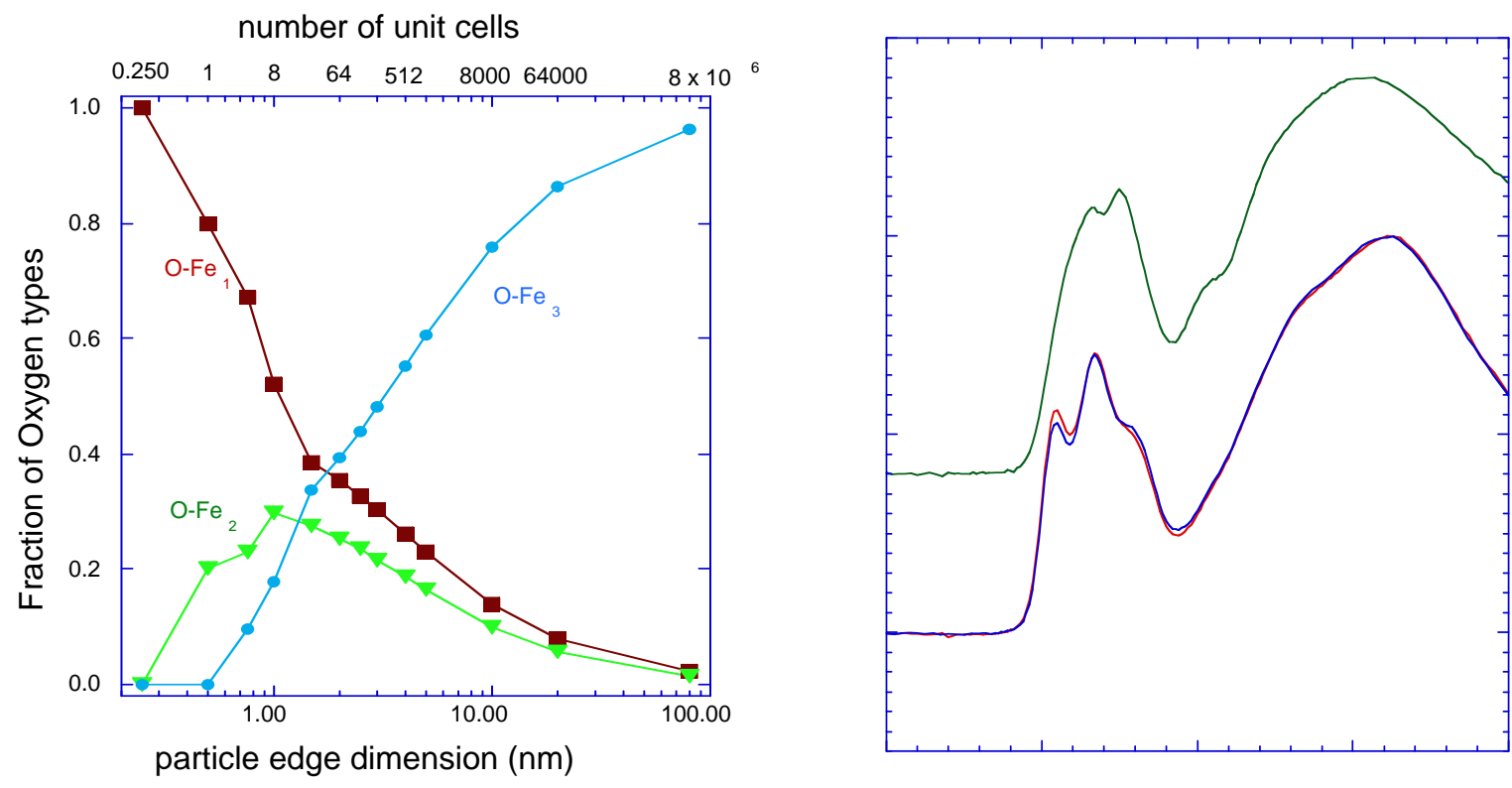

Figure 5. Left: relative numbers of types of O-Fe coordination environments in nanogoethite crystallites based on the ideal structure. Right: Differences in the x-ray absorption edge for nanogoethite particles of differing size. There is a net band shift of $0.4 \mathrm{eV}$ to higher energy for the smaller nanoparticles. 
relaxation might be expected as with $\mathrm{ZnS}$. A second consideration of nanoparticle nonstoichiometry is shown in Figure 6 where the water-equilibrated (1-102) surface plane structure of hematite is shown (Tanwar et al., 2007). Comparison with the ideal bulk structure shows that there is a missing layer of Fe atoms at the surface, as well as tightly bound water molecules. The missing Fe atom layer effectively creates a slab with FeOOH stoichiometry, so that for small enough nanoparticles large deviations from normal hematite composition ought to occur. Hence both simple termination issues, and site underpopulation may affect stoichiometry and probably electronic structure and reactivity.

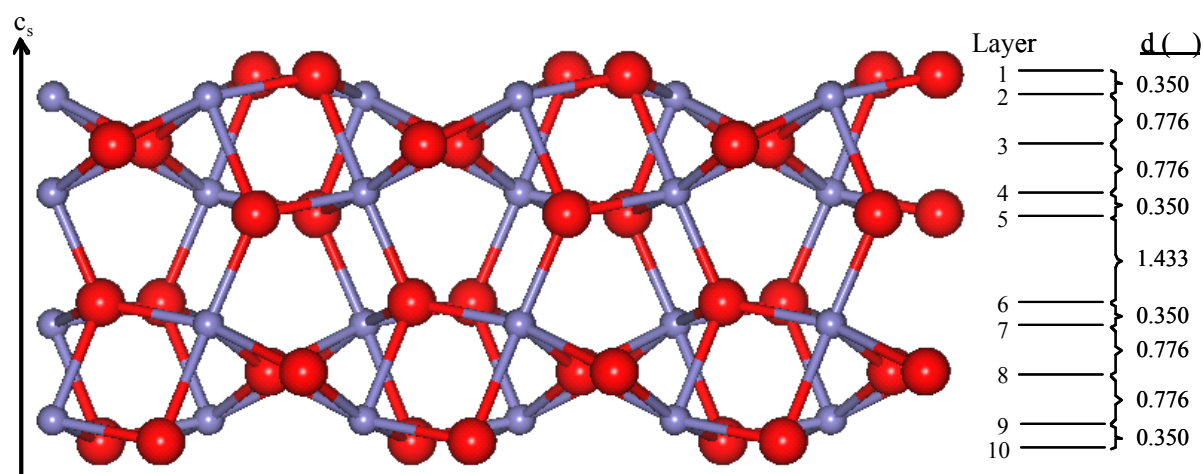

UNUSUAL DEFECT CHEMISTRY AND NANOPHASE STABILITY

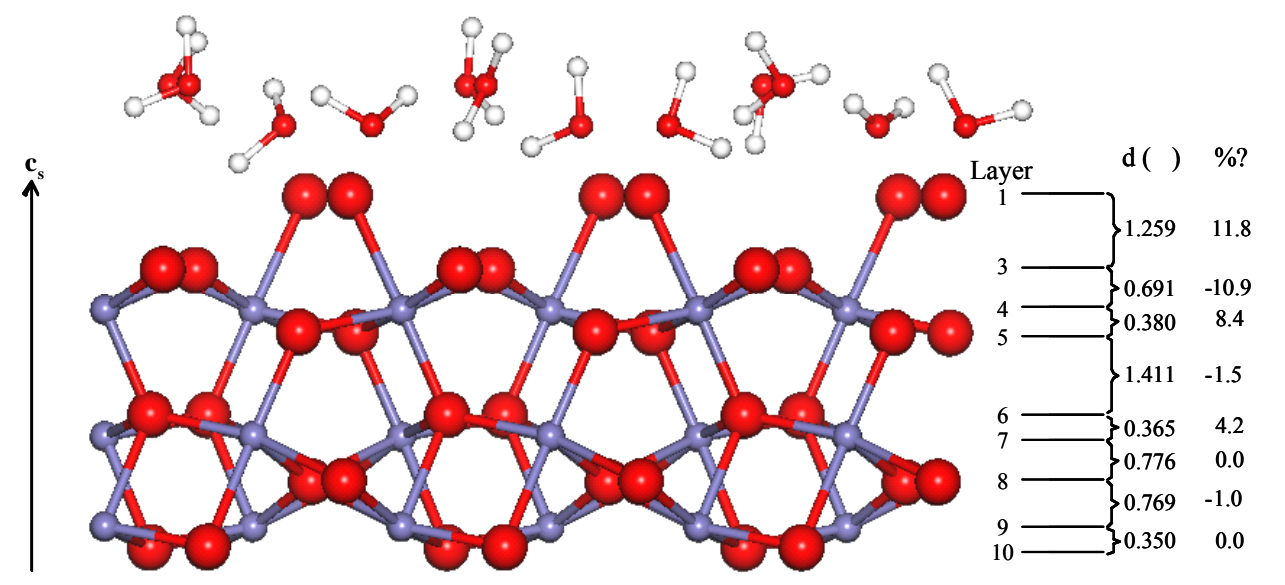

Figure 6.

Bulk structure of (1-102) terminated hematite (top), and the observed surface structure as determined by surface diffraction (below).

Note the missing Fe atomic layer in the observed surface (smaller atoms).

One class of iron oxyhydroxide minerals found almost exclusively only on the nanoscale are the minerals ferrihydrite and schwertmannite. In the case of ferrihydrite the structure has been controversial for years, with different proposed structures and chemistry, some of them actually combinations of mixed individual phases, and a wide range of different x-ray scattering observations. Recently Michel et al. (2006) proposed a structure that appeared to unify most xray scattering observations using a single type of topological motif related to so-called Keggin molecules. The difficulty with ferrihydrite is that it never appears in crystallites larger than about $3 \mathrm{~nm}$, and has stoichiometry varying with size. Hence there is never a typical Bragg diffraction pattern to work from, but rather a diffuse scattering pattern. Accordingly, Michel et al. (2006) tackled the ferrihydrite structure by synchrotron PDF analysis. Although their result 
remains controversial, and there is a lack of quantum calculations available to verify their particular unit cell geometry, the result suggests a natural phase with no larger crystalline analog. Despite seeming unusual, this result could actually be the rule for hydrous nanoparticulate metal hydroxides and oxides, and may explain why so many (largely unsolved) transformations appear to occur during their preparations. Another case of related ilk is schwertmannite, a sulfateoxyhydroxide believed to be related to the akaganeite structure (a hollandite type topology) but also problematic due to small crystallite size, changes in stoichiometry, and changes in bulk chemistry. Past work has shown that the sulfate in schwertmannite, generally considered to be bound tightly in the "tunnels" of the structure, is actually weakly bound probably at the surface of the structure. This type of binding could lead to growth issues that restrict development, resulting in the small crystallites. Another possibility is that the "surface" sulfate is encapsulated in a larger tunnel formed by a series of stacking defects in the structure (figure 7). This could lead to a highly defective structure, but with disorder quite different from the surface poisoned variety. Schwertmannite is associated with acid mine drainage, and is connected with the massive cycling of toxic metals and production of sulfuric acid connected with such sites.

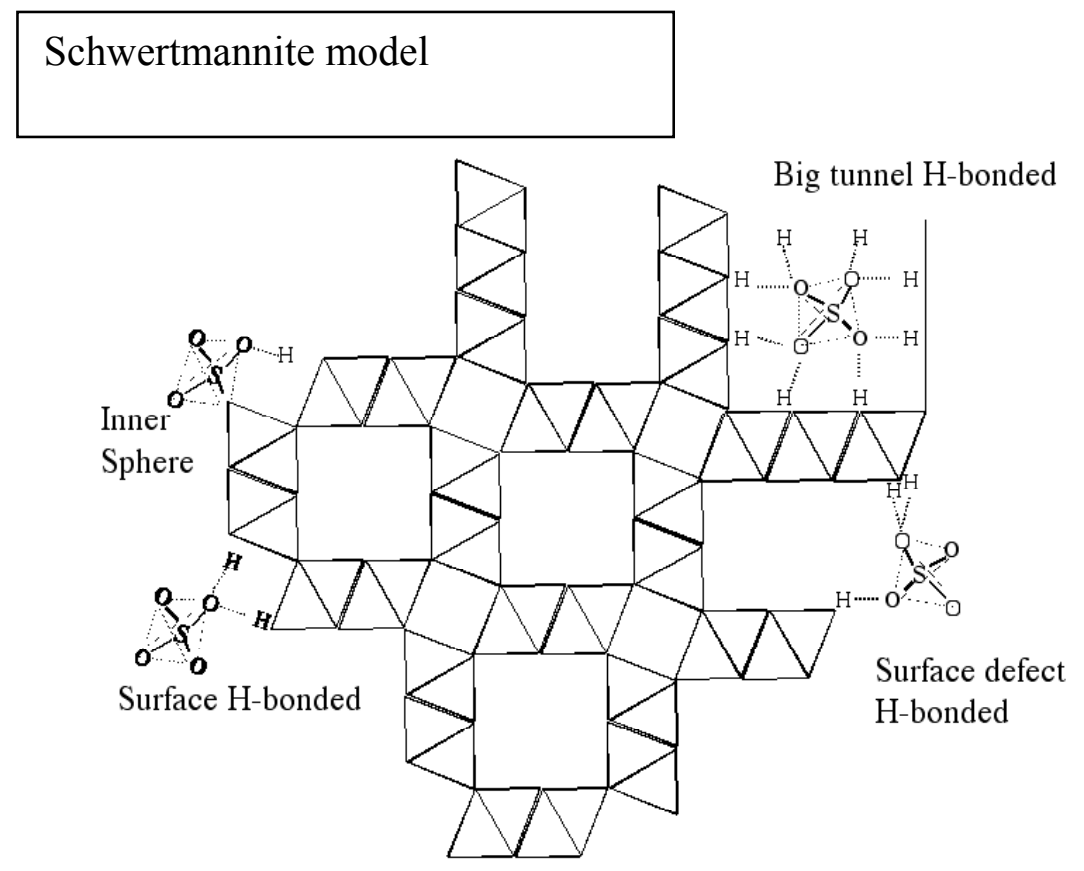

Figure 7. Speculated structure of schwertmannite showing basic backbone (iron oxide octahedral units sharing edges normal to the page) and "tunnel" structure. Hollandite consists of the " 2 × 2 " motif, but a " 3 x 3 " motif seems to be consistent with a larger tunnel occupation by sorbed sulfate (upper right). Hence the defective nature of the phase could be caused by planar stacking faults creating larger tunnels, or by surface-induced disorder. 


\section{ORIENTED AGGREGATION (OA)}

Another feature that is observed in natural nanoparticles, and particularly in Fe oxyhydroxides, is oriented (or crystallographically-oriented) aggregation. In this process, aggregation occurs in such a way that each assembling particle has time to orient itself properly for exact crystallographic attachment. Growth is thus different both in rate and in motif compared to classical "layer by layer" growth. One of the more studied materials demonstrating OA is nanogoethite. Figure 8 shows a series of TEM images representing the process.
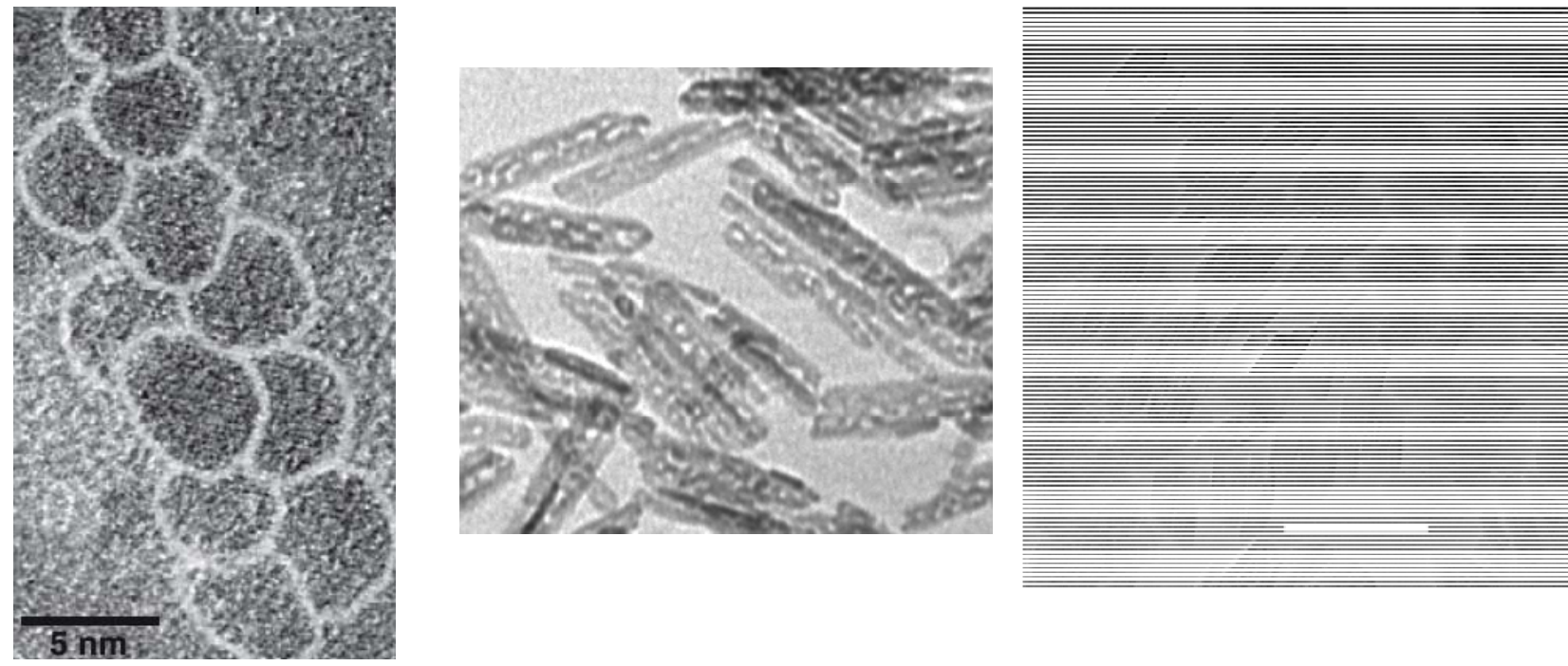

Figure 8. Left to right $\mathrm{OA}$ in nanogoethite. Left: $4 \mathrm{~nm}$ nanoparticles assemble with lattice fringes aligned (from Guyodo et al., 2003) . Center: nanorods of $15 \mathrm{~nm}$ width created by similar OA processes (from Waychunas et al., 2005). Right: Possible OA in aggregation of a different habit of goethite. Note internal contrast fluctuations of 4-5 nm dimension. White bar is $50 \mathrm{~nm}$.

One significant consequence of $\mathrm{OA}$ is the manner in which impurities in the environment might be collected during OA assembly. Whereas normal surface sorption would have complexes that could be removed by changes in fluid properties such as $\mathrm{pH}$, as one may wish to do in a remediation protocol, a toxic complex encapsulated between two OA-joined nanoparticles could not be removed except with dissolution of the particles. Besides this environmental concern, OA presents an interesting subject upon which to understand nanoparticle interaction dynamics in solution, as well as issues important to crystallization such as how solvent molecules react during the necessary desolvation process as the particles come together.

\section{FUTURE WORK}

The work shown here represents a small part of recent and current natural and synthetic analog nanoparticle research at the Nanogeoscience laboratory at LBNL and UC Berkeley. Besides improved structural characterization via $\mathrm{x}$-ray methods, we are also interested in electron transfer processes at nanoparticle surfaces which might be important aspects of microbe-nanoparticle 
interactions, and which may be adopted in some manner for energy-conversion utilization. One additional aspect of this research is the comparison of surface structural results, such as that on large single crystal hematite, with nanoparticle surfaces. It is hoped that by developing a complete understanding of the "crystal chemistry" of mineral surfaces, that this might be profitably applied to nanoparticle surface structures and processes which are so much more difficult to image and refine.

Another important aspect of nanoparticle work relates to the nucleation of nanoparticles at interfaces. In a sense all phases that grow at interfaces from solution have had a regime wherein they had nanoscale dimensions, i.e. essentially the regime of classical nucleation theory. We thus expect that nanoparticle research will also shed light on dissolution/reprecipitation transformations that are particularly important in low temperature geochemistry, as well as on poorly known aspects of heterogeneous nucleation processes.

\section{ACKNOWLEDGEMENTS}

This work was supported by the Director, Office of Science, Office of Basic Energy Sciences, Division of Chemical Sciences, Geosciences, and Biosciences, of the U.S. Department of Energy under Contract No. DE-AC02-05CH11231.

\section{REFERENCES}

[1] Ehrlich, H.L. Geomicrobiology. Marcel Dekker, New York, 1996, 717 p.

[2] Banfield, J.F. and Navrotsky, A. Nanoparticles and the Environment. Rev. in Mineralogy Volume 44, Mineralogical Society of America, 2001349 p.

[3] Gilbert, B., Huang, F., Zhang, H., Waychunas, G.A., Banfield, J.F. Science 2004, 305, 651654.

[4] Huang, F., Gilbert, G., Zhang, H., Banfield, J.F. Phys. Rev. Lett. 2004, 92, 155501

[5] Zhang, H., Chen, B., Yang, R., Waychunas, G.A., Banfield, J.F. (2009, submitted).

[ 6] Navrotsky, A., Mazeina, L., Majzlan, J. Science 2008, 319, 1635-1638.

[7] Tanwar, K.S., Lo, C.S., Eng, P.J., Catalano, J.G., Walko, D.A., Brown, G.E., Waychunas, G.A., Chaka, A.M., Trainor, T.P. Surface Science 2007, 601, 460-474.

[8] Michel, F.M., Ehm, L., Antao, S.M., Lee, P.L., Chupas, P.J., Liu, G., Strongin, D.R., Schoonen, M.A.A., Phillips, B.L., Parise, J.B. Science 2007, 316, 1726-1729.

[9] Guyodo, Y., Mostrom, A., Penn, R.L., Banerjee, S. Geophys. Res. Lett. 2003, 30, 191-194

[10] Waychunas, G.A., Kim. C.S., Banfield, J.F. J. Nanoparticulate Res. 2005, 7, 409-433. 